\title{
Anisotropic nature of the capillary stress tensor
}

\author{
Mojtaba Farahnak ${ }^{1, *}$, Richard Wan ${ }^{1, * *}$, and Mehdi Pouragha ${ }^{2, * * *}$ \\ ${ }^{1}$ Department of Civil Engineering, Schulich School of Engineering, University of Calgary, Canada \\ ${ }^{2}$ Department of Civil and Environmental Engineering, Carleton University, Canada
}

\begin{abstract}
The paper describes a micromechanical approach that explores the anisotropic nature of the capillary stress tensor and its evolution in pendular granular materials via Discrete Element Modeling (DEM) simulations. Dimensionless parameters are used to address the conditions under which the contribution of capillarity (or cohesive interparticle forces) to the stress transmission within a Representative Elementary Volume (REV) is expected to be considerable. From a series of suction-controlled conventional triaxial tests, numerical results show that the significance of the capillary stress and the relative magnitude of its mean to deviatoric components is directly connected to the characteristic particle size and applied stress. In addition, it is shown that the anisotropic character of the capillary stress tensor intensifies with increasing suction. Furthermore, a simple shear test is conducted at constant mean stress to reveal the development of deviatoric capillary stresses in the absence of any change in mean stress, which cannot be captured by the commonly used Bishop's stress expression.
\end{abstract}

\section{Introduction}

Capillarity is a dominant mechanism arising from multiphasic interactions in wet granular materials. The resulting capillary interparticle forces play an important role in the rheology of these materials that are frequently encountered in either nature or engineering problems. Of particular interest, is exploring the various components of stress transmission in partially saturated geomaterials. Furthermore, the long-standing investigation on the question of the existence of an effective stress which would play the same role as Terzaghi's effective stress in the saturated case, implies the significance of this subject matter and its key application in geomechanics.

Attempts to describe the partitioning of stresses in unsaturated soils date back to the pioneering work of Bishop [1] followed by others more recently [2, 3]. On the other hand, complex triphasic interactions have necessitated the use of micromechanical approaches in any study to address the physics operating at the particle scale. Accordingly, the micromechanical analysis of stress transmission describing the effect of capillarity in the pendular regime has been pursued via analytical stress homogenization approach and numerical DEM modeling, see e.g. [4-7].

An important outcome of recent micromechanical studies reveals that capillary forces contribute to both hydrostatic and deviatoric stresses, thereby identifying the drawback of the well-known Bishop's equation in which the capillary stress tensor is spherical. The present study explores in more details the anisotropic nature of the capillary stress tensor using a micromechanical approach. A series of suction-controlled DEM simulations with different loading paths is carried out to highlight the evolution of the deviatoric part of the capillary stress tensor. First, the description of stress in partially saturated granular materials with its equivalent discrete representation is reviewed and implemented in DEM modeling. Subsequently, numerical simulation results are discussed followed by some conclusion remarks.

\section{Stress in triphasic condition}

Bishop [1] proposed a variant of Terzaghi's effective stress expression $\left(\sigma^{\prime}\right)$ for unsaturated condition as the difference between the total stress and a spherical (isotropic) form of contributions from air and water pressures $\left(u_{\mathrm{a}}, u_{\mathrm{w}}\right)$ :

$$
\boldsymbol{\sigma}^{\prime}=\boldsymbol{\Sigma}-\left[u_{\mathrm{a}}-\chi\left(u_{\mathrm{a}}-u_{\mathrm{w}}\right)\right] \boldsymbol{\delta}
$$

where $\delta$ is the Kronecker delta and the parameter $\chi$ is introduced as a scaling coefficient describing the contribution of suction $\left(s=u_{\mathrm{a}}-u_{\mathrm{w}}\right)$. Recently, a more precise micromechanical description of stress in partially saturated condition has been derived by [6] using the homogenization technique in which, for a REV in equilibrium with the applied loads on its boundaries, the total stress is presented as the volume average of stress tensors for the existing phases at the microscale:

$$
\begin{aligned}
\boldsymbol{\Sigma}-u_{\mathrm{a}} \boldsymbol{\delta}= & \frac{1}{V}\left[\sum_{\mathrm{c}} \overrightarrow{f^{c}} \otimes \vec{l}-s\left(V_{\mathrm{w}} \boldsymbol{\delta}+\int_{S_{\mathrm{sw}}} \vec{n} \otimes \vec{x} \mathrm{~d} S\right)\right. \\
& \left.-\gamma\left(\int_{\Gamma} \vec{v} \otimes \vec{x} \mathrm{~d} l+\int_{S_{\mathrm{aw}}}(\boldsymbol{\delta}-\vec{n} \otimes \vec{n}) \mathrm{d} S\right)\right]
\end{aligned}
$$

\footnotetext{
*e-mail: mojtaba.farahnaklang@ucalgary.ca

**e-mail: wan@ucalgary.ca

***e-mail: mehdi.pouragha@carleton.ca

A video is available at https://doi.org/10.48448/7q04-a058
} 
where $\boldsymbol{\Sigma}-u_{\mathrm{a}} \boldsymbol{\delta}$ is the net stress. The first term on the right hand side (RHS) of equation (2) is the well-known Love-Weber stress formula for the so-called contact stress tensor: $\sigma^{\text {cont }}=\frac{1}{V} \sum_{\mathrm{c}} \overrightarrow{f^{\mathrm{c}}} \otimes \vec{l}$, with $\vec{f}^{\mathrm{c}}$ and $\vec{l}$ being the contact force and the branch vector connecting the centers of particles in contact, respectively, and the summation applies over the contacts between particles. The second RHS term of equation (2) is related to the contribution of suction and the arguments in between parentheses are the liquid volume $\left(V_{\mathrm{w}}\right)$ and the action of suction integrated on the particles' wetted surfaces $\left(S_{\mathrm{sw}}\right)$ with normal vector of $\vec{n}$ and position vector of $\vec{x}$. The last term in RHS of equation (2) describes the contribution of surface tension $(\gamma)$ acting over the contact line $(\Gamma)$ with $\vec{v}$ being the inward co-normal vector to the interface; along with the membrane-like stress $\boldsymbol{\pi}_{\mathrm{aw}}=-\gamma(\boldsymbol{\delta}-\vec{n} \otimes \vec{n})$ within airwater interface. The capillary stress tensor is then defined by considering the second and third terms in a form of generalized stress description $\sigma^{\text {cap }}=-\chi\left(u_{\mathrm{a}}-u_{\mathrm{w}}\right)-\boldsymbol{B}$.

The anisotropy (non-spherical form) of the capillary stress tensor emerges clearly from this formulation in terms of the distribution of pendular liquid bridges within the assembly. Interestingly, it has been numerically shown [6] that the capillary stress tensor is equivalent to a discrete representation of the capillary interparticle forces in a quasi-static condition:

$$
\sigma^{\mathrm{cap}}=\frac{1}{V} \sum_{\mathrm{m}} \vec{f}^{\mathrm{cap}} \otimes \vec{l}
$$

where $\vec{f}^{\text {cap }}$ is the capillary force, and the summation applies to the all menisci (m) formed between particles. Such a stress description can be readily calculated in a DEM modeling in which the magnitude of the capillary force can be obtained by solving the Young-Laplace equation with respect to the properties of liquid bridges:

$$
\left|\vec{f}_{i}^{\mathrm{cap}}\right|=\pi R_{i} \sin \delta_{i}\left(s R_{i} \sin \delta_{i}+2 \gamma \sin \left(\delta_{i}+\theta\right)\right)
$$

with $R_{i=1,2}$ being the radius of two interacting particles, $\delta$ and $\theta$ being the half filling and contact angles, respectively.

\section{DEM simulations}

DEM numerical simulations are herein carried out to highlight the anisotropic nature of the capillary stress tensor in pendular regime. The open-source YADE code [8] has been used for the simulation of suction-controlled loading paths for a REV consisting of spherical particles. The wet condition is applied assuming a uniform suction through the assembly under thermodynamic equilibrium. As such, a liquid bridge connects a pair of particles if the solution for the Young-Laplace equation exists, i.e., just before the rupture distance. Accordingly, at every time step during the simulation, the interparticle capillary forces are computed with respect to the imposed suction. In addition, the DEM simulations are implemented under constant suction during both the isotropic and deviatoric loading steps. The required information for the capillary effect, i.e., the liquid bridge properties are provided by a previously implemented finite difference approach solving the Young-Laplace equation [9].

The mechanical interactions between solid particles are characterized by the classical linear elastic-plastic contact laws that are described by a local Coulombian friction coefficient $\mu=\tan (\phi)$ and two ratios $k_{\mathrm{n}} / \bar{D}$ and $k_{\mathrm{t}} / k_{\mathrm{n}}$ representing the local elastic stiffness with respect to the average particle diameter $\bar{D}$, see Table 1 .

Two series of DEM modeling simulations are considered. First, conventional triaxial test is incorporated to investigate the significance of capillary stress contribution to the total net stress with respect to key dimensionless parameters. Next, a simple shear test is carried out to demonstrate the effect of stress path on the development of deviatoric capillary stress under constant mean stress.

Table 1. Discrete element method model parameters

\begin{tabular}{ccccc}
$\begin{array}{c}\tan (\phi) \\
(-)\end{array}$ & $\begin{array}{c}k_{\mathrm{n}} / \bar{D} \\
(\mathrm{MPa})\end{array}$ & $\begin{array}{c}k_{\mathrm{t}} / k_{\mathrm{n}} \\
(-)\end{array}$ & $\begin{array}{c}\gamma \\
(\mathrm{N} / \mathrm{m})\end{array}$ & $\begin{array}{c}\theta \\
\left(^{\circ}\right)\end{array}$ \\
\hline 0.5 & 10 & 1 & 0.073 & 0 \\
\hline
\end{tabular}

\section{Results and discussions}

\subsection{Conventional triaxial tests}

DEM numerical simulations begin with isotropic compression of an assembly of 10000 spherical particles within a rectangular parallelepiped until reaching the desired confining pressure. A very low interparticle friction is adopted to obtain a relatively dense packing state. The isotropic samples are first prepared under dry condition and then capillary effects, i.e., the cohesive interactions, are introduced with respect to a prescribed suction before applying the deviatoric loading. Herein, the effects of capillarity on the preparation of samples, which usually result in looser packing states, are neglected. Such a sample preparation procedure helps us generate several assemblies with very close initial microstructural conditions. This aspect is crucial for the objective comparisons of the results of subsequent loading paths.

Subsequent to the isotropic compression, the deviatoric loading (under constant suction) is applied in vertical direction while the stress is fixed in lateral directions. During all loading steps in the simulations, the quasi-static condition is constantly checked against a very low level of inertial number or unbalanced force ratio.

In order to analyze the evolution of capillary stress during triaxial loading condition and its relative magnitude to the applied and contact stresses, we incorporate the following key micromechanical dimensionless parameters for wet condition:

$$
\kappa_{\mathrm{t}}=\frac{p\langle r\rangle}{k}, \quad \kappa_{\mathrm{c}}=\frac{\left\langle f^{\mathrm{n}}\right\rangle}{k\langle r\rangle}, \quad \kappa_{\mathrm{r}}=\frac{\pi \gamma}{k}, \quad \kappa_{\mathrm{s}}=\frac{s\langle r\rangle}{\gamma}
$$

with $k$ being the contact normal stiffness, $p$ being the mean stress, $\langle r\rangle$ and $\left\langle f^{\mathrm{n}}\right\rangle$ are average radius and mechanical contact normal force, respectively. These 
parameters have been previously described in details in [10]. The parameters $\kappa_{\mathrm{t}}$ and $\kappa_{\mathrm{c}}$ are related to the applied stress at boundaries and the mechanical contact forces, respectively. As opposed to the dry case, these two parameters are not equivalent in the wet condition. On the other hand, $\kappa_{\mathrm{r}}$ and $\kappa_{\mathrm{s}}$ are connected to the capillary mechanism. Depending on the loading path, the $\kappa_{\mathrm{t}}$ parameter might change during the course of loading. Consequently, $\kappa_{\mathrm{c}}$ evolves in response to the applied loading condition and in the presence of capillary interactions. It is worth mentioning that the ratio $\kappa_{\mathrm{t}} / \kappa_{\mathrm{r}}$ resembles the parameter that has been defined as the reduced pressure $\left(P^{*}=P D / \pi \gamma\right)$ by [7]. The ratio $\kappa_{\mathrm{t}} / \kappa_{\mathrm{r}}$ scales the intensity of the applied stress to the cohesive interparticle forces.

Herein, we examine the contribution of capillary stress to the mechanical behavior of wet granular media. To this end, we evaluate the effect of cohesive interactions with respect to the size of particles, confining pressure, and suction value using the defined dimensionless parameters.

For the first series of simulations, the contribution of capillary stress to the stress transmission within the REV is investigated with respect to the size of particles and the applied confining pressure. In this regard, two ranges of particle sizes are considered with average radii of $\langle r\rangle=0.1 \mathrm{~mm}$ and $0.01 \mathrm{~mm}$. The particle size distribution is chosen to be uniform with a maximum to minimum particle size ratio of 1.5 . The chosen parameters correspond to ratios of $\kappa_{\mathrm{t}_{0}} / \kappa_{\mathrm{r}}=4.2,0.42$, and 0.042, while $\kappa_{\mathrm{t}_{0}}=10^{-3}$ and $10^{-4}$ address the effect of confining pressure $\left(p_{0}\right)$. It must be noted that while $\kappa_{\mathrm{t}_{0}}$ is a controlling parameter for the applied stress condition, $\kappa_{\mathrm{c}}$ evolves during the triaxial test as explained earlier. Considering a pendular saturation condition, $\kappa_{\mathrm{S}}=13.7$ is kept constant for these simulations, the effect of which is demonstrated in the next step. The parameters are presented in Table 2.

Results of the triaxial loading tests are shown in figures 1 and 2, in which the evolutions of mean and deviatoric components of the capillary stress tensor are illustrated. The magnitude of the capillary stresses are normalized by the contact stress for a meaningful comparison of their contributions to the mechanical response. Figure 1 indicates that the intensity of mean capillary stress depends on ratio $\kappa_{\mathrm{t}_{0}} / \kappa_{\mathrm{r}}$, where a drastic decrease of its contribution to the mean stress is observed by increasing the size of particles and the applied confining pressure. Such an observation is directly related to the change in relative magnitude of the cohesive interactions, i.e., the capillary forces with respect to the size of particles and in comparison to the mechanical contact forces in response to the external loading. On the other hand, the relatively high contribution of the capillary stress in the post-peak behavior, as contact stress decreases, results into a larger difference in the ratio $p^{\text {cap }} / p^{\text {cont }}$ between the peak and the critical state.

Figure 2 shows the evolution of deviatoric capillary stress which is consistent with the evolution of fabric anisotropy of the liquid bridges during deviatoric loading, as previously illustrated by [10]. In a same vein, the relative magnitude of the deviatoric component of the capillary stress tensor increases with decreasing $\kappa_{\mathrm{t}_{0}} / \kappa_{\mathrm{r}}$ ratio.

Table 2. Dimensionless parameters for triaxial tests

\begin{tabular}{cccc} 
& $\kappa_{\mathrm{t}_{0}}$ & $\kappa_{\mathrm{t}_{0}} / \kappa_{\mathrm{r}}$ & $\kappa_{\mathrm{s}}$ \\
\hline Triaxial test 1 & $10^{-3}$ & 4.2 & 13.7 \\
\hline Triaxial test 2 & $10^{-3}$ & 0.42 & 13.7 \\
\hline Triaxial test 3 & $10^{-4}$ & 0.042 & 13.7 \\
\hline
\end{tabular}

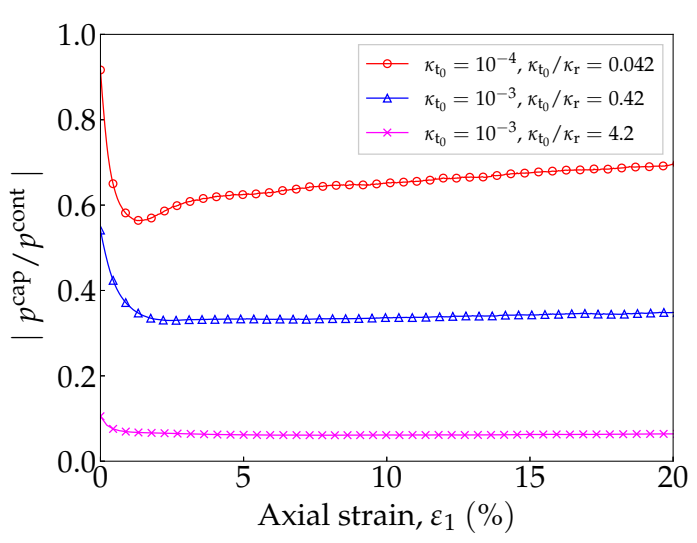

Figure 1. The evolution of mean capillary stress during DEM triaxial tests.

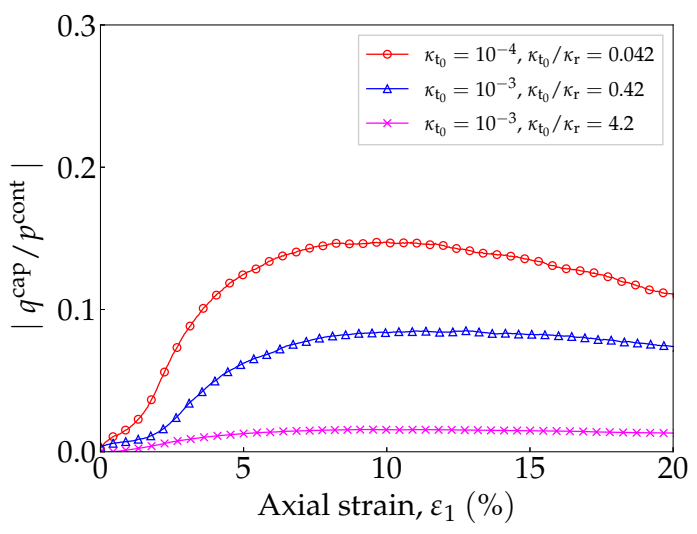

Figure 2. The evolution of deviatoric capillary stress during DEM triaxial tests.

Next, the effect of suction magnitude is evaluated considering three different values of $\kappa_{\mathrm{S}}=6.85,13.7$, and 20.5; while keeping $\kappa_{\mathrm{t}_{0}}=10^{-4}$ and $\kappa_{\mathrm{t}_{0}} / \kappa_{\mathrm{r}}=0.042$ constant for all of the three cases. The $\kappa_{\mathrm{S}}$ values are selected such that the condition of isolated liquid bridges in pendular saturation regime is maintained.

Figure 3 illustrates the evolution of anisotropic character of the capillary stress tensor with respect to the different values of $\kappa_{\mathrm{s}}$. Results show that the intensity of suction also determines the deviatoric behavior of the capillary effect and the relative magnitude of deviatoric capillary stress. Accordingly, the higher value of the suction results in a more pronounced anisotropic behavior for the capillary stress. In this regard, figure 4 indicates that such an observation is connected to the effect of suction on the deviatoric component of the capillary 
stress arising from distant interactions $\left(q^{\text {dcap }}\right)$, i.e., liquid bridges forming between non-contacting particles. In fact, the rupture of the liquid bridges during rearrangement and relative movement of the particles, depends on the intensity of the suction, with a delay in its occurrence at lower suctions.

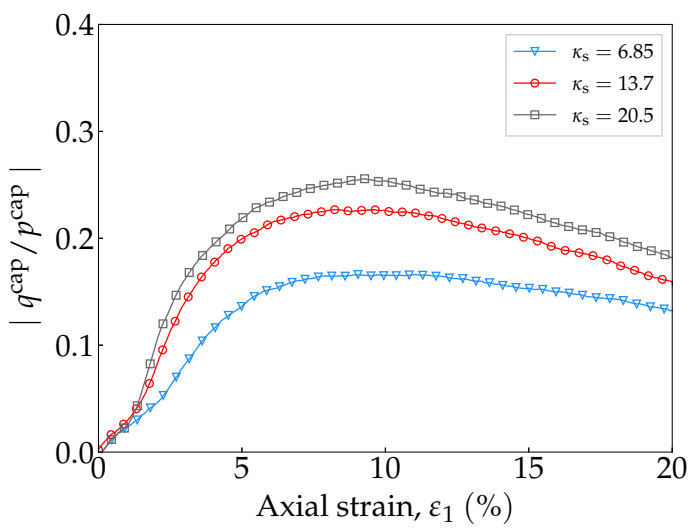

Figure 3. The effect of $\kappa_{\mathrm{s}}$ on the evolution of deviatoric behavior of the capillary stress during DEM triaxial tests.

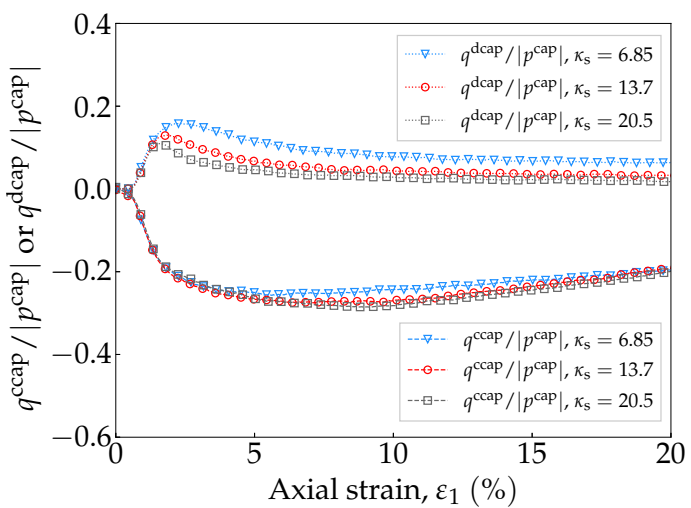

Figure 4. Decomposition of the deviatoric capillary stress for contact (ccap) and distant (dcap) interactions.

\subsection{Simple shear test}

In order to explore further the anisotropic nature of the capillary stress tensor and the ability of the micromechanical approach to capture this characteristic feature, a unique stress path is incorporated in this section. In this regard, a DEM simulation of $p$-constant simple shear test is carried out to monitor the evolution of deviatoric capillary stress. For this purpose, the simple shear loading $\left(\partial v_{1} / \partial x_{2}=\right.$ const., $\left.\Sigma_{11}=\Sigma_{22}=\Sigma_{33}\right)$ is applied on an isotropically confined sample using periodic boundary condition. Figure 5 shows that in spite of keeping the mean pressure $(p)$ constant, the deviatoric component of the capillary stress tensor develops during shearing of the sample. It must be noted that such an anisotropic feature of capillary (suction) stress cannot be captured using Bishop' equation in which the capillary stress is assumed to have a spherical form.

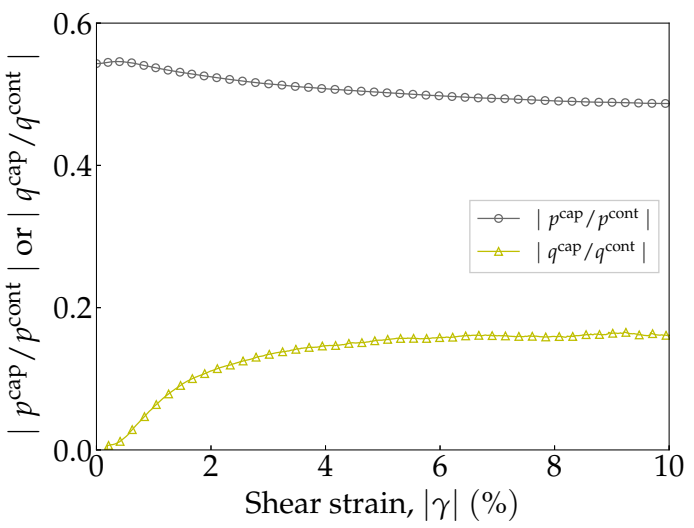

Figure 5. The evolution of capillary stress components during DEM simple shear test $\left(\kappa_{\mathrm{t}_{0}}=10^{-3}, \kappa_{\mathrm{t}_{0}} / \kappa_{\mathrm{r}}=0.42, \kappa_{\mathrm{s}}=13.7\right)$.

\section{Conclusions}

A micromechanical approach has been presented to evaluate the contribution of capillary stress to the mechanical response of wet granular materials and its anisotropic nature. Various dimensionless parameters are incorporated to address the significance of the capillary stress contribution relative to the applied loading condition. Results from DEM suction-controlled triaxial test simulations have shown that both mean and deviatoric component of the capillary stress become considerable when the ratio $\kappa_{\mathrm{t}_{0}} / \kappa_{\mathrm{r}}$ decreases. Such a condition corresponds to the smaller particle size ranges and lower confining stresses. In addition, the deviatoric character of the capillary stress tensor intensifies with increasing $\kappa_{\mathrm{S}}$ parameter, i.e., with increasing suction.

Finally, the anisotropic nature of the capillary stress tensor is investigated during a $p$-constant simple shear loading that sustains deviatoric capillary stresses, thanks to the micromechanical approach.

\section{References}

[1] A. Bishop, Tek. Ukebl. 106, 859 (1959)

[2] M. Nuth, L. Laloui, Int. J. Numer. Anal. Met. 32, 771 (2008)

[3] C. Zhang, N. Lu, J. Eng. Mech. 146, 04019135 (2020)

[4] V. Richefeu, F. Radjai, M.S. El Youssoufi, Eur. Phys. J. E 21, 359 (2006)

[5] L. Scholtès, B. Chareyre, F. Nicot, F. Darve, Int. J. Eng. Sci. 47, 64 (2009)

[6] J. Duriez, R. Wan, J. Eng. Mech. 142, 04016099 (2016)

[7] V.D. Than, S. Khamseh, A.M. Tang, J.M. Pereira, F. Chevoir, J.N. Roux, J. Eng. Mech. 143, C4016001 (2017)

[8] V. Šmilauer et al., Yade Documentation 2nd ed. (The Yade Project, 2015)

[9] J. Duriez, R. Wan, Acta Geotech. 12, 67 (2017)

[10] M. Pouragha, R. Wan, J. Duriez, N.H. Sultan, Int. J. Numer. Anal. Met. 42, 1935 (2018) 